\title{
Open Electronic Experiment System based on MCU Programmer Design Keli Yuan ${ }^{1}$, Xingshan $\mathrm{Li}^{1, \text { a }}$, Jian $\mathrm{Lu}^{2}$ \\ ${ }^{1}$ Luohe medical college, Luohe, 462000, China \\ ${ }^{2}$ Henan industrial vocational and technical college, Nanyang, 473000, China \\ aEmail:604141388@qq.com
}

Keywords: Single-chipmicrocomputer, Full development board, Programming adapter, Programming adapter interface module

\begin{abstract}
This paper describes the design of the programmer, function of the practical programmer was introduced, and presents the chip type of the programmer can support. The study includes the innovative design for imaging electronics course teaching experimental device, object oriented higher vocational technical secondary school, technical school, teachers and students. The results of the project to take a most intuitive and the most feasible way to replace the existing teaching equipment, so that with the use of learning, at any time to use, and can meet the requirements of the students after school.
\end{abstract}

\section{Introduction}

\section{FX-A full development board function.}

The FX-A development board is composed of two major parts, which are the software experimentation area and the hardware experimentation area. The software is used to develop various kinds of application projects quickly with various functional modules and components. Experimental development board as shown in Figure 1 below.

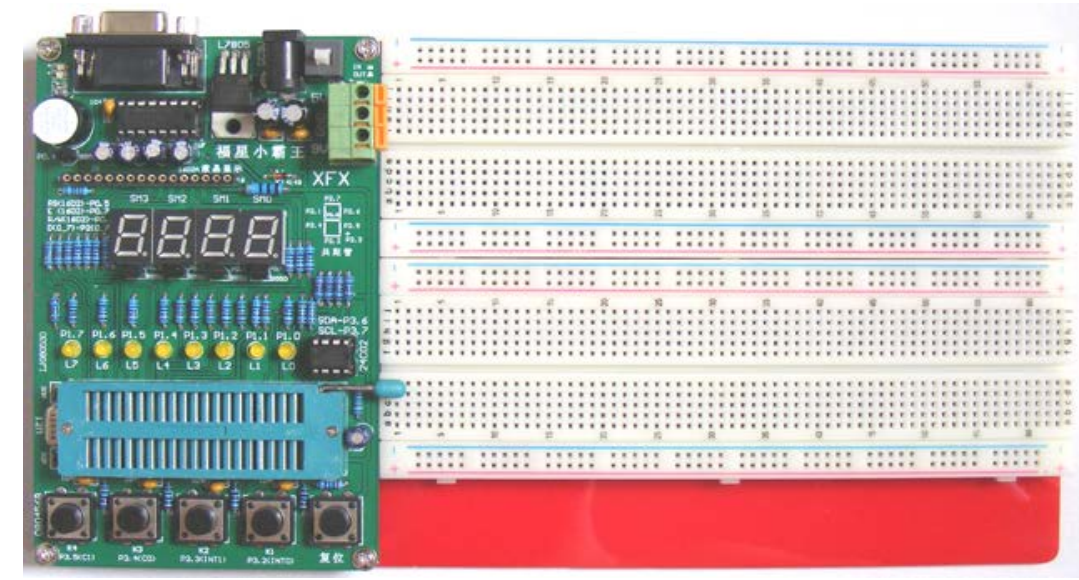

Fig 1 FX-A full development board

AT89S52 and AT89S51 was used in development board for the experimental development of the chip, there are 2 digital tube, 8 light-emitting diodes, four button switches, a simple audio circuit, RS232 serial communication interface, serial memory, liquid crystal display interface are installed on the board. As shown in Figure 1 [1]. The use of this experimental board can not only water lights, buzzer, LED dynamic scanning, interrupt, counters and other basic programming practice, but also can learn I2C interface, LCD display technology and PC serial communication is very popular at present.

At the same time for more convenient for beginners to understand the controlled object, in the experimental board screen printing surface shows that the various control points of the pin symbols, user-friendly rapid development of control procedures on the experimental board.

Development board software experimentation area is based on the students to learn microcontroller technology experimental requirements to develop a multi-function circuit board, which able to complete as many as more than ten kinds of experiments and portfolio projects. And 
with ISP online programming function as shown in Figure 2.

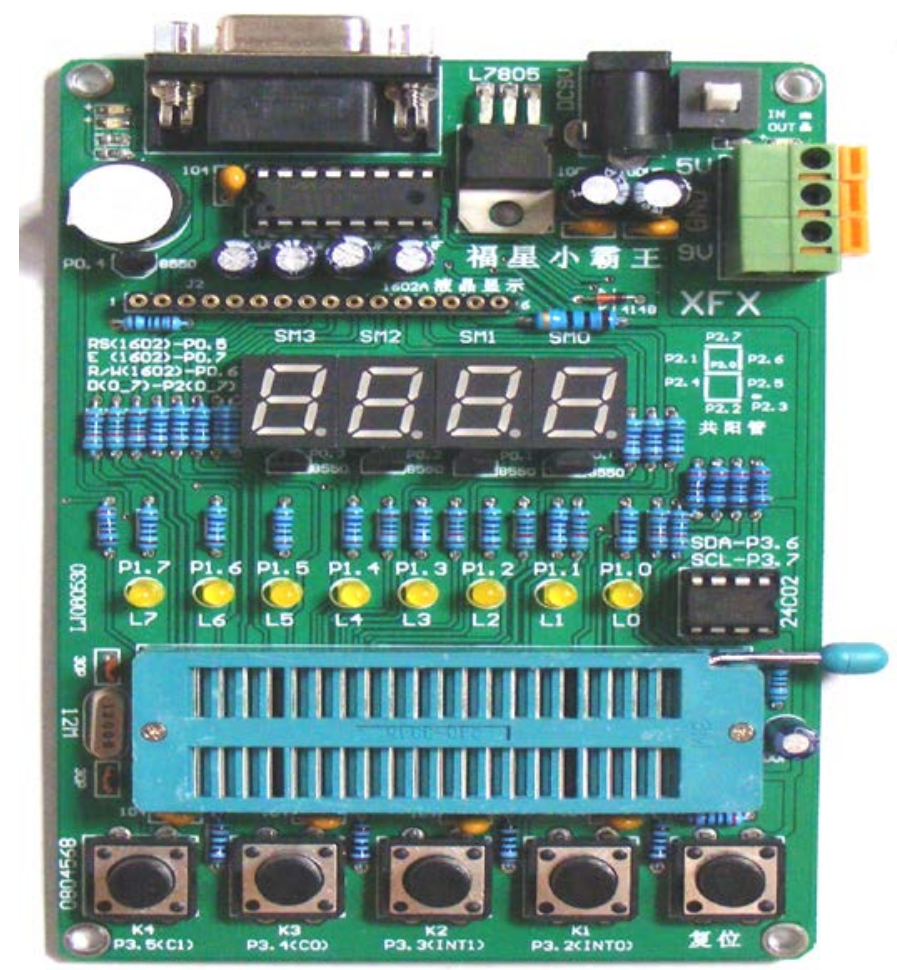

Fig2 FX-A software experimental area

\section{Purpose of design}

Fuxing group project programmer is designed for beginners microcontroller programmer practical design and development, is an indispensable set of open electronic components in the experimental system. The programmer can burn ATMEL series single chip, with stable performance, burning speed, cost advantages.

\section{Supported chip models}

The most classic at present and AT89C51, C52, C55 produced by the ATMEL company of market share of the largest,and the latest S51, S52,AT89C1051,2051,4051 and other chips. Especially suitable for eager to learn 51 microcontroller and want to try to reduce the input of learning for beginners. After all, the 51 series has become the industry standard, 51 MCU learning, so that everything becomes intelligent under the control of MCU, which is a dream lover and fancier[2]. 89C51 single-chip microcomputer is the current market share first, Million pieces the size of a large number of production of the 51 series chip were produced by Winbond company in every day,which is fully compatible with AT company,so the students don't have to worry about its source. AT company to withdraw from the production to the production of S51 series is only in order to prevent the loss. As shown in the following figure. 


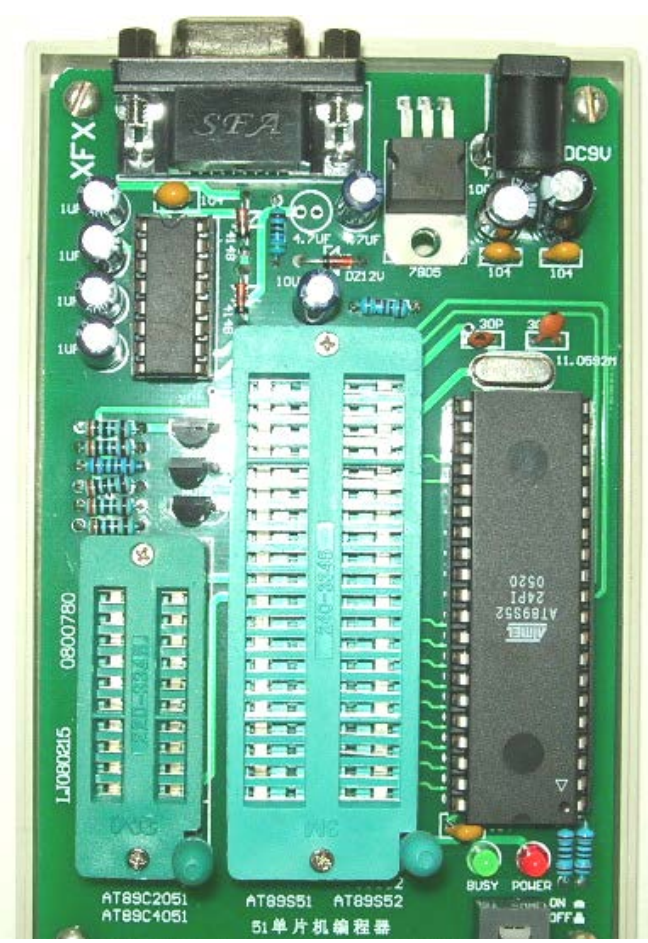

Fig3 Fuxing programmer

\section{Technical indicators}

Fusion programmer working for $9 \mathrm{v}$ dc voltage, programmer working voltage is dc $5 \mathrm{v}$ circuit board, through the $\mathrm{L} 7805$ voltage regulator, the work is less than 0.5 watts. The boost circuit is designed by using MAX232, and the circuit structure is simple and stable. Up to $12 \mathrm{v}$ to $5 \mathrm{v}$ voltage, meets the need of individual chip $12 \mathrm{v}$ programming requirements. Serial port and programmer form a complete set of nine core thread and an external power supply, the programmer work circuit with a power switch and lamp, plastic floor make integral effect is more beautiful.

The function design of the monitoring chip is one of the core technology of the programmer, programming for all operations are required by the control chip to complete scheduling. We use AT89S52 chip to monitor the chip, with low cost, easy to develop and so on. In the monitoring chip we designed a communication protocol with the computer, the programmer hardware function has been fully demonstrated, the independent intellectual property rights for our future on the function of the programmer provides technical support to upgrade[3].

Another programmer to use the specific performance as follows.

1. Using a serial port communication, erasing, burning in the process of programming, calibration various operating completely controlled by monitoring chip on the programmer, not affected by PC configuration and its main frequency, so the burning rate is high, can reach $100 \%$, burning speed and burning speed has nothing to do with the grade of the microcomputer.

2. With 57600 baud rate, high speed data transmission, programming can speed and general parallel programming was comparable to that after the test, write a $4 \mathrm{k}$ ROM burning AT89S51 need only $9.5 \mathrm{~S}$, and read and check for only $3.5 \mathrm{~S}$.

3. Small size, the external power adapter to the programmer to provide $9 \mathrm{v}$ power supply, the greatest degree of protection the user computer, power switch and convenient user when not in use do not have to make the programmer charged for a long time. Very much in line with the requirements of beginners to learn $51 \mathrm{MCU}[4]$.

4. Software friendly interface, menus, toolbars, shortcut keys is complete, the entire Chinese operations, provide encryption function, can protect your creative property rights.Can be said to be small but complete.

5. Function is perfect, with programming, read, blank check, inspection, erase, encryption, etc.

6. 40pin and 20pin lock socket, all devices with all the first foot alignment, no additional jumper 
for DIP chip without any adapter.

7. Made of high quality universal lock socket, and such problems as poor contact thoroughly to say goodbye, can burn write 40 feet microcontroller chip microcontroller chips and 20 feet.

8. Because the use of 9 pin communication, so that it will not be a printer and grab a print port, want to burn at any time, so that the chip programming to become a happy.

\section{Hardware connection}

1. Good communication cable connected to the programmer.

2. Insert the serial port plug into the computer serial port.

3. The 9V DC power supply and the programmer connected, power switch is pressed, the programmer of LED light, that the power supply is switched on.

4. Then you can install the software, the software supports Win9x/me / 2000 / NT, standard Windows interface.

As shown in the figure below:

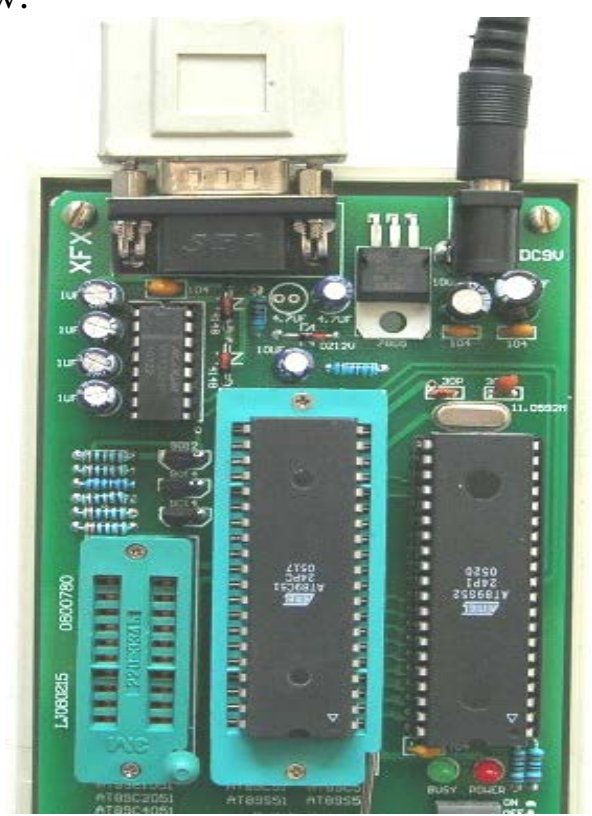

Fig4 Programmer hardware connection

\section{Programmer accessories}

Fuxing programmer, programmer All Suite should host the serial data line, 9V DC power supply and software driver CD.

\section{Function and structure of programming adapter}

Programming adapter is an important feature of experimental platform, the adapter can cooperate FX - A full analysis of single-chip microcomputer development board to complete the programming and simulation work, main operation program of record, to read, calibration, encryption, erasing and single-chip microcomputer control, real-time operation, stop, and other functions[5]. The appearance of the adapter and the structure as shown in the figure below: 


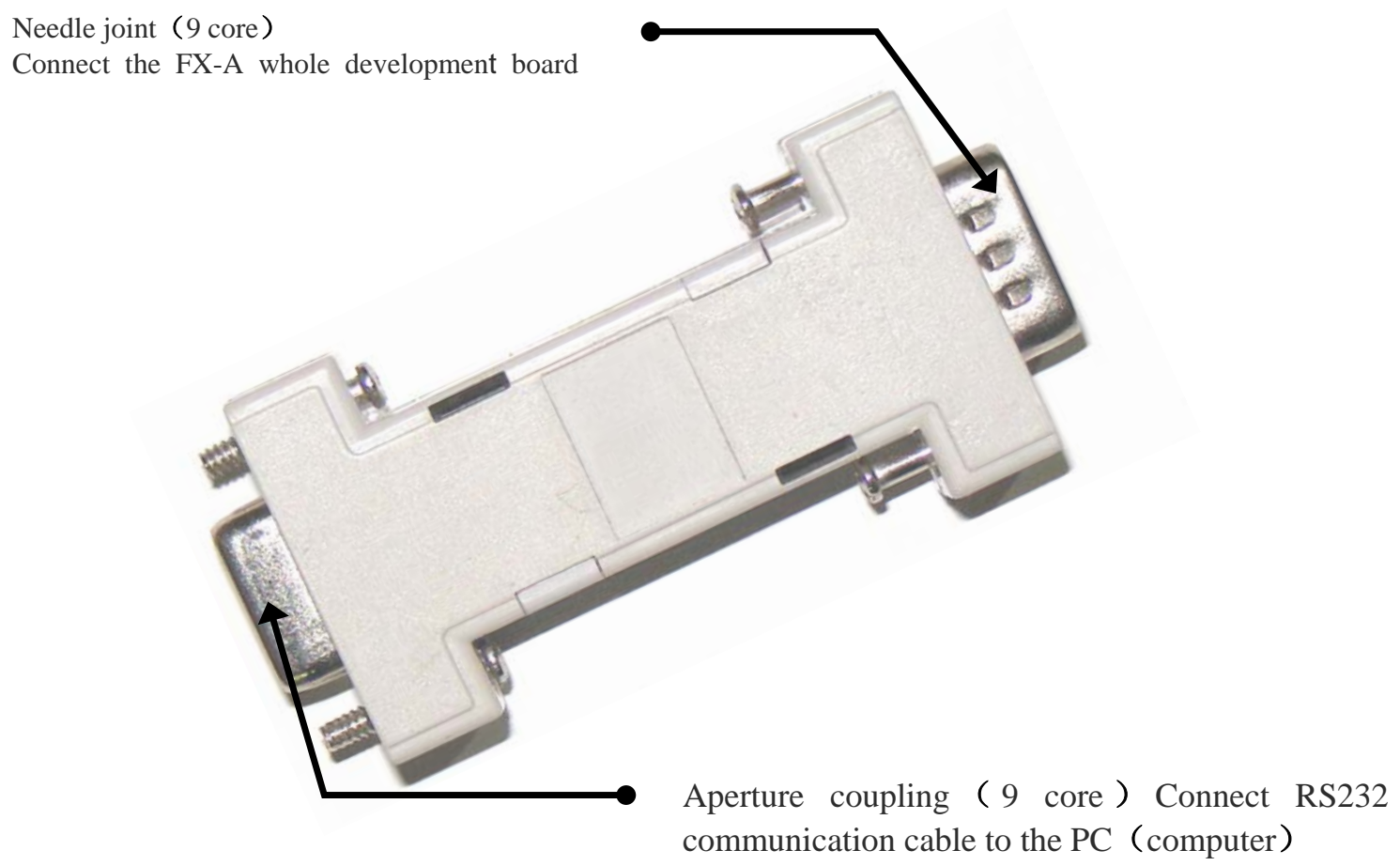

Fig5 The programming adapter

\section{Conclusion}

After the experiment of third party testing instrument supporting the design, programming adapter module circuit the test parameters of open electronic experiment system have reached the initial design requirements, and set aside other system upgrade interface module and upgrade space. System for the operation of the experimental circuit to achieve intelligent programming control, stable operation, can achieve the desired parameters and the expected purpose, the design of the system security and reliability greatly improved.

\section{Acknowledgement}

In this paper, the research was sponsored by the education technology and equipment and practice education research project topics in Henan Province In 2015 (Project No. GZS112).

\section{Reference}

[1] Guoyan Duan.Research and development of electronic technology virtual experiment system.[D]. Chongqing:Southwest Jiaotong University, 2013.

[2] Chunyu Cheng,Zhenyu Wu,Yanan Wu,et al. Development of analog electronic technology experiment platform for hierarchical teaching mode.Laboratory Science[J].2014,17(5):

[3] Xinyu Zhu.The development and application of electronic design automation experiment system [D]:Beijing: Beijing university of posts and telecommunications,2012

[4] Lei Su. Development and application of the separated electrical experiment device. Electronic design engineering [J].2011,9:138-140.

[5] Shisheng Yan,Chengyao Zhong,Liefeng Wang. Research and Development of New Analog Electronics Technology Experimental Device.Journal og Hainan Normal University:Natural Science[J].2012,25(3). 